\title{
Spectral method for matching exterior and interior elliptic problems
}

\author{
Piotr Boronski \\ Center for Turbulence Research \\ Stanford University, Bldg. 500, Stanford, CA 94305-3035 \\ e-mail: boronski@gmail.com
}

\begin{abstract}
A spectral method is described for solving coupled elliptic problems on an interior and an exterior domain. The method is formulated and tested on the two-dimensional interior Poisson and exterior Laplace problems, whose solutions and their normal derivatives are required to be continuous across the interface. A complete basis of homogeneous solutions for the interior and exterior regions, corresponding to all possible Dirichlet boundary values at the interface, are calculated in a preprocessing step. This basis is used to construct the influence matrix which serves to transform the coupled boundary conditions into conditions on the interior problem. Chebyshev approximations are used to represent both the interior solutions and the boundary values. A standard Chebyshev spectral method is used to calculate the interior solutions. The exterior harmonic solutions are calculated as the convolution of the free-space Green's function with a surface density; this surface density is itself the solution to an integral equation which has an analytic solution when the boundary values are given as a Chebyshev expansion. Properties of Chebyshev approximations insure that the basis of exterior harmonic functions represents the external near-boundary solutions uniformly. The method is tested by calculating the electrostatic potential resulting from charge distributions in a rectangle. The resulting influence matrix is well-conditioned and solutions converge exponentially as the resolution is increased. The generalization of this approach to three-dimensional problems is discussed, in particular the magnetohydrodynamic equations in a finite cylindrical domain surrounded by a vacuum.
\end{abstract}

Key words: influence matrix, spectral method, Chebyshev polynomials, boundary integral method, magnetohydrodynamics, Green's functions, harmonic functions, Laplace's equation, exterior problem

\section{Motivation}

The search for a self-sustaining magnetohydrodyamic dynamo has taken on great momentum in recent years, as researchers have sought to produce dynamos in the 
laboratory [1.2/345] and in simulations [6/78|9|10/11/12|13|15|16]. One of the fundamental problems in numerical magnetohydrodynamics is the formulation of boundary conditions. The governing equations describe the velocity and magnetic field in in a finite container of electrically conducting fluid. At the container boundaries, the velocity is specified, but the magnetic field is not. Instead, the magnetic field is required to satisfy continuity conditions with the exterior magnetic field in the domain surrounding the fluid. The nature of these conditions depends on the properties of the surrounding medium; a complete discussion can be found in [17].

Under the quasi-static approximation [17], for a given velocity field $\mathbf{u}$ and magnetic Reynolds number $R m$, the equations describing the interior magnetic field are

$$
\begin{aligned}
\partial_{t} \mathbf{B} & =\nabla \times(\mathbf{u} \times \mathbf{B})+\frac{1}{R m} \Delta \mathbf{B} \\
\nabla \cdot \mathbf{B} & =0
\end{aligned}
$$

The case of a fluid of finite electric conductivity restricted to a finite volume and surrounded by vacuum is of special importance because it models a number of experimental, geophysical, and astrophysical configurations. Since there are no electrical currents in a vacuum, $\mathbf{B}^{v a c}$ is curl-free, and is therefore the gradient of a potential if the exterior domain is simply connected. The exterior magnetic field then obeys:

$$
\begin{aligned}
\mathbf{B}^{\mathrm{vac}} & =\nabla \phi^{\mathrm{vac}} \\
\Delta \phi^{\mathrm{vac}} & =0 \\
\mathbf{B}^{\mathrm{vac}} & \rightarrow 0 \quad|\mathbf{x}| \rightarrow \infty
\end{aligned}
$$

but is otherwise not fixed. The magnetic field is required to be continuous at the boundary:

$$
\mathbf{B}-\mathbf{B}^{\mathrm{vac}}=0 \quad \mathbf{x} \in \partial \Omega
$$

In this case, continuity of all three components of the magnetic field are sufficient to uniquely determine both the interior and exterior fields. Our ultimate goal is to transform (2)-(3) into boundary conditions that can be applied to (11) without calculating $\mathbf{B}^{\mathrm{vac}}$. The general principle we will employ is to construct a complete basis of exterior solutions $\mathbf{B}^{\mathrm{vac}}$ of (2) in a preprocessing step, and to calculate $\left.\mathbf{B}\right|_{\partial \Omega}$ for each member of the basis. The matching conditions (3) will then yield boundary conditions for $\mathbf{B}$.

To explore this approach, we will apply it to the simpler analogous scalar problem of the Poisson problem in an interior domain. We will require the solution to match continuously to an exterior solution satisfying Laplace's equation. In this case, both Dirichlet and Neumann matching conditions are necessary to specify a unique solution. Formally, we wish to solve the following problem: 


$$
\begin{aligned}
& \Delta \Phi=\rho \quad \text { in } \Omega \\
& \Delta \phi=0 \quad \text { outside } \Omega
\end{aligned}
$$

with boundary conditions:

$$
\begin{aligned}
\Phi(\mathbf{x})-\phi(\mathbf{x})=0 & & \mathbf{x} \in \partial \Omega \\
\partial_{n} \Phi(\mathbf{x})-\partial_{n} \phi(\mathbf{x})=0 & & \mathbf{x} \in \partial \Omega \\
\nabla \phi(\mathbf{x}) \rightarrow 0 & & |\mathbf{x}| \rightarrow \infty
\end{aligned}
$$

where $\Omega$ is a bounded domain with boundary $\partial \Omega$. A physical interpretation of (4)(5) is that of an electrostatic potential $\Phi$ of a field generated by charges distributed in space with the density $-\rho$, where the electrical permeability of the vacuum is taken to be one by the choice of units. We wish to calculate the interior solution $\Phi$ without explicitly constructing $\phi$.

There exists a vast literature on the numerical solution of the fundamental physical problems (4)-(5) and (1)-(3). We will briefly survey a small portion of this literature here, and postpone a more detailed comparison between our method and others to a later section.

The main tool by which exterior domains can be eliminated is Green's theorem, which replaces elliptic differential equations over a domain with integrals over the bounding surfaces. The use of methods based on boundary integrals has grown explosively since the 1970s-1980s to solve engineering problems from fields such as acoustics, elasticity, electromagnetism and fluid mechanics $\left[\begin{array}{ll|l|l|l|l|l|l|l|l|l|l|l|l|}18 & 19 & 20 & 21 & 22 & 23 & 24 & 25 & 26\end{array}\right]$. Hybrid methods, coupling a differential equation formulation in a domain and a boundary integral formulation at the boundary via an influence matrix, were also developed at the same time to solve (4)-(5) and similar problems. The majority of these approaches have been based on finite elements and are hence applicable to complicated real-world geometries. The boundary integrals are discretized with techniques derived from finite element theory, leading to the term boundary element method, and the hybrid methods use finite elements to solve the equations in the domain.

This situation contrasts with magnetohydrodynamics, which has been dominated by spectral methods. Spherical domains are standard, for geophysical and astrophysical reasons. Spectral methods can then be based on spherical harmonics and the poloidal-toroidal decomposition [7/8,910]. The solution to Laplace's equation on the exterior is immediate and, moreover, solutions and associated boundary conditions for each spherical harmonic and toroidal or poloidal component are decoupled. Boundary conditions at the interface can then be formulated for each mode without the use of an influence matrix.

The technique which we will describe is based on spectral methods, but the geometry is assumed to be somewhat more complicated. Our technique occupies the niche 
which spectral methods staked out in the 1970s-1980s when the use of Chebyshev polynomials became commonplace [27,28] to represent domains with one or more non-periodic directions. In keeping with this tradition, we expect its main application to be to tensor-product domains whose boundaries consist of a small number of piecewise-smooth surfaces, such as the finite three-dimensional cylinder which is our eventual goal.

We also mention here some other recent approaches to solving the magnetohydrodynamic equations [12:13,14 15 16], with a view to generalizing the geometry and/or increasing parallelization. In [13 14], a finite volume method is used to discretize the solution in the interior, which is matched to that in the exterior vacuum via a boundary element method. [15] describes an integral equation formulation for the entire domain, and [16] uses finite elements with a penalty method to apply boundary conditions.

\section{Influence matrix formulation}

We formulate a two-stage method for solving (4)-(5), consisting of an initial preprocessing step which depends only on the geometry, followed by a step whereby solutions for many different distributions $\rho$ can be generated at little incremental cost. This is the usual description of the decomposition of $\Phi$ into homogeneous and particular solutions, with the additional proviso that solutions in the exterior domain are to be taken into account in the preparation of the homogeneous solutions. We will construct the homogeneous solutions by generating harmonic bases $\left\{\Phi_{j}^{h}\right\}$ and $\left\{\phi_{j}\right\}$ of interior and exterior solutions, corresponding to Dirichlet boundary data $\left\{f_{j}\right\}$ to be specified later. We decompose (4)-(5) into the Poisson and Laplace problems:

$$
\begin{array}{lll}
\Delta \Phi^{p}=\rho \text { in } \Omega & \Delta \Phi_{j}^{h}=0 \text { in } \Omega & \Delta \phi_{j}=0 \text { outside } \Omega \\
\left.\Phi^{p}\right|_{\partial \Omega}=0 & \left.\Phi_{j}^{h}\right|_{\partial \Omega}=f_{j} & \left.\phi_{j}\right|_{\partial \Omega}=f_{j} \\
& \left.\nabla \phi_{j}\right|_{\infty}=0
\end{array}
$$

and then construct the linear superpositions:

$$
\Phi=\Phi^{p}+\Phi^{h} \quad \Phi^{h}=\sum_{j} c_{j} \Phi_{j}^{h} \quad \phi=\sum_{j} c_{j} \phi_{j}
$$

Then 


$$
\begin{aligned}
& \Delta \Phi=\quad \Delta \Phi^{p}+\sum_{j} c_{j} \Delta \Phi_{j}^{h}=\rho \quad \text { in } \Omega \\
& \Delta \phi=\quad \sum_{j} c_{j} \Delta \phi_{j}=0 \quad \text { outside } \Omega \\
& \left.(\Phi-\phi)\right|_{\partial \Omega}=\left.\Phi^{p}\right|_{\partial \Omega}+\left.\sum_{j} c_{j}\left(\Phi_{j}^{h}-\phi_{j}\right)\right|_{\partial \Omega}=0 \\
& \left.\nabla \phi\right|_{\infty}=\left.\quad \sum_{j} c_{j} \nabla \phi_{j}\right|_{\infty}=0
\end{aligned}
$$

are already satisfied by construction, while

$$
\left.\partial_{n}(\Phi-\phi)\right|_{\partial \Omega}=\left.\partial_{n} \Phi^{p}\right|_{\partial \Omega}+\left.\sum_{j} c_{j} \partial_{n}\left(\Phi_{j}^{h}-\phi_{j}\right)\right|_{\partial \Omega}=0
$$

constitutes a system of equations to be solved for $c_{j}$, where the derivative with respect to the normal is taken in the direction from the interior to the exterior region for both $\Phi$ and $\phi . \Phi$ is then set equal to the sum in (7). If the interior harmonic functions are not stored, $\Phi$ can be obtained by solving:

$$
\begin{aligned}
\Delta \Phi & =\rho \text { in } \Omega \\
\left.\Phi\right|_{\partial \Omega} & =\sum_{j} c_{j} f_{j}
\end{aligned}
$$

Using $\mathbf{x}_{i}$ to index points on the boundary, (12) can be discretized as:

$$
\sum_{j}\left[\partial_{n}\left(\phi_{j}-\Phi_{j}^{h}\right)\left(\mathbf{x}_{i}\right)\right] c_{j}=\partial_{n} \Phi^{p}\left(\mathbf{x}_{i}\right)
$$

Equation (15) shows that the goal of the preprocessing step is the construction and inversion of the influence or capacitance matrix:

$$
C_{i j} \equiv\left[\partial_{n}\left(\phi_{j}-\Phi_{j}^{h}\right)\left(\mathbf{x}_{i}\right)\right]
$$

The functions $\left\{f_{j}\right\}$ are required to constitute a complete set for values along the discretized boundary $\partial \Omega$. Another way to describe the influence matrix is as a discrete representation of the difference between the Dirichlet-to-Neumann mappings in the exterior and in the interior regions.

Equivalently, $\left.\partial_{n}\left(\phi_{j}-\Phi_{j}^{h}\right)\right|_{\partial \Omega}$ can be represented as coefficients of a basis set $\left\{g_{i}\right\}$ (which may be identical with the set of boundary value functions $\left\{f_{i}\right\}$ ) along each boundary. Equation (12) is then discretized as:

$$
\sum_{j}\left\langle\partial_{n}\left(\phi_{j}-\Phi_{j}^{h}\right), g_{i}\right\rangle c_{j}=\left\langle\partial_{n} \Phi^{p}, g_{i}\right\rangle
$$

Although we will use $\mathbf{x}_{i}$ and the notation in (15) in what follows, the method is easily reformulated using (17). 


\section{Solution of Poisson and Laplace problems}

\subsection{Interior domain}

We now turn to the solution of (6). For the interior problems listed in the first two columns, we assume that we dispose of a solver able to compute solutions to Poisson's equation in $\Omega$ with any specified boundary values. In principle, any numerical method can be used. In our particular case, we use a spectral discretization [28]

$$
\Phi(x, y)=\sum_{k, l=0}^{K, L} \mathcal{T}_{k}(x / H) \mathcal{T}_{l}(y)
$$

for the rectangle $[-H, H] \times[-1,1]$. The spectral basis functions are the Chebyshev polynomials $\mathcal{T}_{k}(x)=\cos (k \arccos (x))$. Taking $H \geq 1$, we set $K \geq L$. We use a standard method [28] to solve the Poisson equation with Dirichlet boundary conditions, diagonalizing the discretized second derivative operator in $y$, and using recursion relations to treat the second derivative in $x$.

\subsection{Exterior harmonic functions}

Our main focus is on the construction of the exterior harmonic solutions, specified in the third column of (6). In order to avoid truncating or spatially discretizing the exterior domain, we will construct $\left\{\phi_{j}\right\}$ using the fundamental solution of the Laplace equation: the Green's function satisfying

$$
\begin{array}{rlrl}
\Delta_{\mathbf{x}^{\prime}} G\left(\mathbf{x} ; \mathbf{x}^{\prime}\right) & =\delta\left(\mathbf{x}-\mathbf{x}^{\prime}\right) & \\
\nabla G\left(\mathbf{x} ; \mathbf{x}^{\prime}\right) & =0 \quad 0 \quad \text { for } \mathbf{x}^{\prime} \rightarrow \infty
\end{array}
$$

For a specified boundary value distribution $f(\mathbf{x})$, we first calculate an appropriate source distribution $\sigma(\mathbf{x})$ on the boundary by solving the integral equation:

$$
\int_{\partial \Omega} G\left(\mathbf{x} ; \mathbf{x}^{\prime}\right) \sigma\left(\mathbf{x}^{\prime}\right)=f(\mathbf{x}) \quad \text { for } \mathbf{x} \in \partial \Omega
$$

The exterior harmonic function $\phi(\mathbf{x})$ required is then:

$$
\phi(\mathbf{x}) \equiv \int_{\partial \Omega} G\left(\mathbf{x} ; \mathbf{x}^{\prime}\right) \sigma\left(\mathbf{x}^{\prime}\right)
$$

where $\mathbf{x}$ takes values either on or off $\partial \Omega$.

We now apply (21)-(22) to our particular test problem of a rectangle. We divide the set of boundary distributions into four sets, each taking non-zero values on only one 
side of the rectangle. In two dimensions, the fundamental Green's function solving (20) is

$$
\frac{-1}{2 \pi} \ln \left|\mathbf{x}-\mathbf{x}^{\prime}\right|
$$

Equation (21) thus reduces to:

$$
\int_{a}^{b} \frac{-1}{2 \pi} \ln \left|x-x^{\prime}\right| \sigma\left(x^{\prime}\right) d x^{\prime}=f(x)
$$

Equation (24) is known as Symm's or Carleman's equation and has the following solution [2930]:

$$
\begin{array}{r}
\sigma(x)=\frac{-2}{\pi \sqrt{(x-a)(b-x)}}\left[\int_{a}^{b} \frac{\sqrt{\left(x^{\prime}-a\right)\left(b-x^{\prime}\right)} f^{\prime}\left(x^{\prime}\right) d x^{\prime}}{x^{\prime}-x}\right. \\
\left.+\frac{1}{\ln ((b-a) / 4)} \int_{a}^{b} \frac{f\left(x^{\prime}\right) d x^{\prime}}{\sqrt{\left(x^{\prime}-a\right)\left(b-x^{\prime}\right)}}\right]
\end{array}
$$

if $b-a \neq 4$. (If $b-a=4$ then the second integral in (25) can be replaced by an arbitrary constant if $\int_{a}^{b} f(t)[(t-a)(b-t)]^{-\frac{1}{2}} d t=0$.)

Up to now, we have not specified the Dirichlet boundary values $f$. The choice of boundary value distributions is restricted only by the requirement, stated in section 2. that the set of distributions form a basis for functions defined on the boundary $\partial \Omega$. Because we use Chebyshev polynomials to represent the interior solutions, it is convenient to take as boundary values $f_{k}(x)$ each of the functions $\mathcal{T}_{k}(x / H)$ on the interval $[-H, H]$. The corresponding solutions $\sigma_{k}(x ; H)$ obtained from evaluating (25) are:

$$
\sigma_{k}(x ; H)=A_{k} \frac{\mathcal{T}_{k}(x / H)}{\pi H \sqrt{1-\left(\frac{x}{H}\right)^{2}}} \quad ; \quad A_{k}= \begin{cases}2 \pi k & k>0 \\ -2 \pi[\ln (H / 2)]^{-1} & k=0\end{cases}
$$

This remarkable property - the fact that that weighted Chebyshev polynomials are also obtained as the source distributions $\sigma_{k}(x)$ when the boundary values $f_{k}(x)$ are Chebyshev polynomials - is related to the very reason that Chebyshev polynomials are optimal in approximating polynomials on the interval. The function $1 / \pi \sqrt{1-x^{2}}$ in (26) (for $H=1$ ) is the weight with respect to which Chebyshev polynomials are orthogonal on the interval and is the asymptotic density of the Chebyshev interpolation points $\cos (\pi j / J)$, the extrema of the Chebyshev polynomials. See [31.32] for further details. Note also that the orthogonality of the Chebyshev polynomials with respect to this weight causes the second integral in (25) to vanish except for $\mathcal{T}_{0}$. 
The corresponding harmonic functions $\phi_{k}^{x}$ are constructed via

$$
\phi_{k}^{x}(\mathbf{x})=\int_{-H}^{H} \frac{-1}{2 \pi} \ln \left|\mathbf{x}-x^{\prime} \hat{\mathbf{e}}_{x}\right| \sigma_{k}\left(x^{\prime} ; H\right) d x^{\prime}
$$

and are illustrated in figure 1 . Specifying values along along the segment $[-1,1]$ in the $y$ direction, we obtain:

$$
\phi_{l}^{y}(\mathbf{x})=\int_{-1}^{1} \frac{-1}{2 \pi} \ln \left|\mathbf{x}-y^{\prime} \hat{\mathbf{e}}_{y}\right| \sigma_{l}\left(y^{\prime} ; 1\right) d y^{\prime}
$$

Note that $\sigma_{k}(x ; H)=\sigma_{k}(x / H ; 1) / H$ for $k>0$ and $\sigma_{0}(x ; H)=\frac{\ln (1 / 2)}{H \ln (H / 2)} \sigma_{0}(x / H ; 1)$.

The harmonic functions corresponding to specified values along the lower or upper boundaries $(y= \pm 1,|x|<H)$ are $\phi_{k}^{x}\left(\mathbf{x} \mp 1 \hat{\mathbf{e}}_{y}\right)$; those corresponding to the left or right boundaries $(x= \pm H,|y|<1)$ are $\phi_{l}^{y}\left(\mathbf{x} \mp H \hat{\mathbf{e}}_{x}\right)$. We do not require the functions $\phi_{k}^{x}, \phi_{l}^{y}$ either inside or outside $\Omega$, but only the values and normal derivatives on the boundary. Although, for example, the values of $\phi_{k}^{x}\left(\mathbf{x}+1 \hat{\mathbf{e}}_{y}\right)$ on the lower boundary are merely the specified values $f_{k}(x)$, its values on the other three boundaries must be calculated via (27). When evaluating the normal derivatives, the kernel $G\left(\mathbf{x} ; x^{\prime}\right)$ is differentiated before integration:

$$
\begin{aligned}
\partial_{y} \phi_{k}^{x}(x, y) & =\partial_{y} \int_{-H}^{H} \frac{-1}{2 \pi} \ln \sqrt{\left(x-x^{\prime}\right)^{2}+y^{2}} \sigma_{k}\left(x^{\prime} ; H\right) d x^{\prime} \\
& =\int_{-H}^{H} \frac{-1}{2 \pi} \frac{y}{\left(x-x^{\prime}\right)^{2}+y^{2}} \sigma_{k}\left(x^{\prime} ; H\right) d x^{\prime}
\end{aligned}
$$

and similarly for $\partial_{x} \phi_{l}^{y}$.

Any exterior harmonic function can be approximated by the truncated series:

$$
\begin{aligned}
\phi^{K, L}(\mathbf{x})= & \sum_{k=0}^{K-1}\left[c_{k}^{x,-} \phi_{k}^{x}\left(\mathbf{x}+H \hat{\mathbf{e}}_{x}\right)+c_{k}^{x,+} \phi_{k}^{x}\left(\mathbf{x}-H \hat{\mathbf{e}}_{x}\right)\right] \\
& +\sum_{l=0}^{L-1}\left[c_{l}^{y,-} \phi_{l}^{y}\left(\mathbf{x}+1 \hat{\mathbf{e}}_{y}\right)+c_{l}^{y,+} \phi_{l}^{y}\left(\mathbf{x}-1 \hat{\mathbf{e}}_{y}\right)\right]
\end{aligned}
$$

The potential $\phi(\mathbf{x})$ of (30) is defined by the $2(K+L)$ coefficients $\left\{c_{k}^{x,-}, c_{k}^{x,+}, c_{l}^{y,-}, c_{l}^{y,+}\right\}$. A very important property of the harmonic basis $\left\{\phi_{k}^{x}(\mathbf{x}), \phi_{l}^{y}(\mathbf{x})\right\}$ is that it represents a near-boundary field uniformly. This means that the truncated series (30) converges uniformly for any smooth boundary data and for all locations $\mathrm{x}$ near the boundary. This is a direct consequence of the excellent convergence properties of Chebyshev approximation applied to (24); a proof can be found in [33]. This property does not necessarily hold for other harmonic bases, in particular spherical harmonics, for which near-boundary convergence cannot be achieved, leading to a strong Gibbs effect. 
In the taxonomy of boundary integral methods, equations (21) and (22) constitute an indirect method, in that the intermediate surface charge density $\sigma$ is constructed; this is done by solving the Fredholm integral equation of the first kind (24). The surface charge density is a single-layer rather than a double-layer (dipole) potential; equivalently $G$, rather than $\partial G / \partial n$, is used in the representation. Because the method determines only the boundary values and normal derivatives of the exterior solution, it is not the preferred approach when the exterior potential is itself required at each time step: although the exterior solution can be sampled at any location, this is computationally expensive, as is often the case for boundary integral methods.
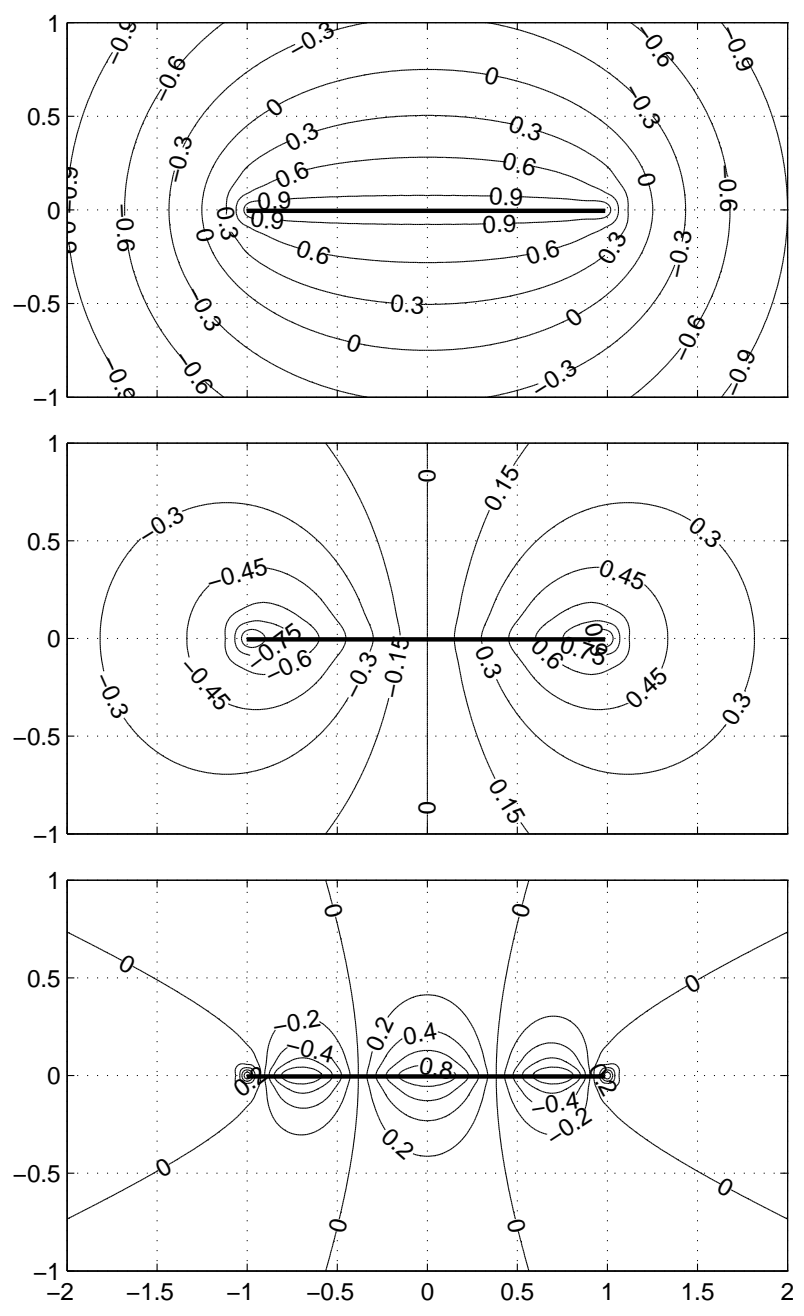

Fig. 1. Potentials $\phi_{k}^{x}(\mathbf{x})$ generated by line source distributions $\sigma_{k}(x ; H=2)$. Values of the potentials on the line segment $x \in[-1,1]$ correspond to the Chebyshev polynomials $\mathcal{T}_{k}(x)$. From top to bottom: $\sigma_{0}(x), \sigma_{1}(x), \sigma_{4}(x)$. 


\section{Electrostatic example}

We apply our method to a simple problem from electrostatics, the distribution of electric charges $-\rho_{m}$ confined in a rectangular domain but localized around the origin:

$$
\rho_{m}(r, \theta)= \begin{cases}r^{m} e^{-r^{2} / \delta^{2}} \cos (m \theta) & \text { for }|x| \leq 1 \text { and }|y| \leq 1 \\ 0 & \text { for }|x|>1 \text { or }|y|>1\end{cases}
$$

The $r^{m}$ factor in (31) ensures regularity of $\rho_{m}$ at $r=0$. The potential $\tilde{\Phi}_{m}$ due to unbounded sources (not restricted to the interior domain) can be found analytically:

$$
\begin{aligned}
& \tilde{\Phi}_{m=0}(r, \theta)=\frac{\delta^{2}}{4}\left[E i\left(1, \frac{r^{2}}{\delta^{2}}\right)+2 \log (r)\right] \\
& \tilde{\Phi}_{m=1}(r, \theta)=\frac{\delta^{4}}{4 r}\left[e^{-\frac{r^{2}}{\delta^{2}}}-1\right] \cos \theta \\
& \tilde{\Phi}_{m=2}(r, \theta)=\frac{\delta^{4}}{4 r^{2}}\left[\left(\delta^{2}+r^{2}\right) e^{-\frac{r^{2}}{\delta^{2}}}-\delta^{2}\right] \cos 2 \theta
\end{aligned}
$$

where $\operatorname{Ei}(a, \lambda)$ is the error function $\operatorname{Ei}(a, \lambda) \equiv \int_{1}^{\infty} e^{-\lambda r} r^{-a} d r$ and $\tilde{\Phi}_{m}$ are chosen to be finite at $r=0$.

We seek the corresponding electric potential. The parameter $\delta$ is chosen to make $\rho_{m}$ very small near the boundaries. We expect the solution to be almost unaffected by the presence of boundaries. The source distribution $\rho_{m=0}(r, \theta)$ should therefore lead to a potential which is almost axisymmetric. Figure 2 shows the potential obtained numerically for $\delta^{2}=0.15$ using the spectral resolution $N=8$ in both directions. The domain boundary is represented by a bold square. The contours are almost perfectly circular, as should be the case for $\delta$ small, showing that the presence of the boundaries has minimal effect.

To evaluate the error convergence of the method we computed the relative error $E_{m}(N)$ defined as

$$
E_{m}(N)=\sup _{r, \theta} \frac{\left|\tilde{\Phi}_{m}(r, \theta)-\Phi_{m}^{N}(r, \theta)\right|}{\left|\Phi_{m}(r, \theta)\right|}
$$

where $\Phi_{m}^{N}(r, \theta)$ is the solution computed numerically with spectral resolution $N$ in both spatial directions of the bounding square and $\tilde{\Phi}_{m}(r, \theta)$ is the analytic solution (32) in the absence of the bounding square. Figure 3 proves the exponential convergence of the method.

Figures 4-5 show the electric potentials $\Phi_{m=1}^{N=16}$ and $\Phi_{m=2}^{N=16}$ for $\delta=0.1$. Figure 6 shows $\Phi_{m=1}^{N=16}$ with $\delta=2$. In figure 7, the dipole source distribution has been 


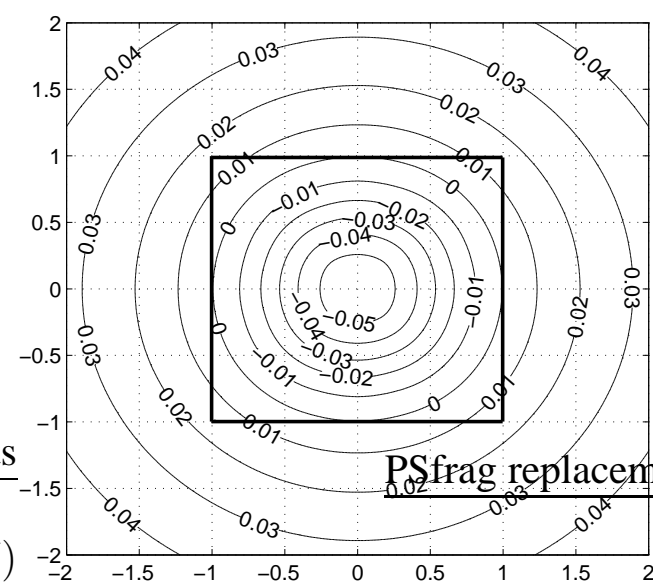

Fig. 2. Potential $\Phi_{m=0}^{N=8}$ for $\delta=0.15$. Maximal relative error is $E_{m=0}(N=8) \approx 0.03$.

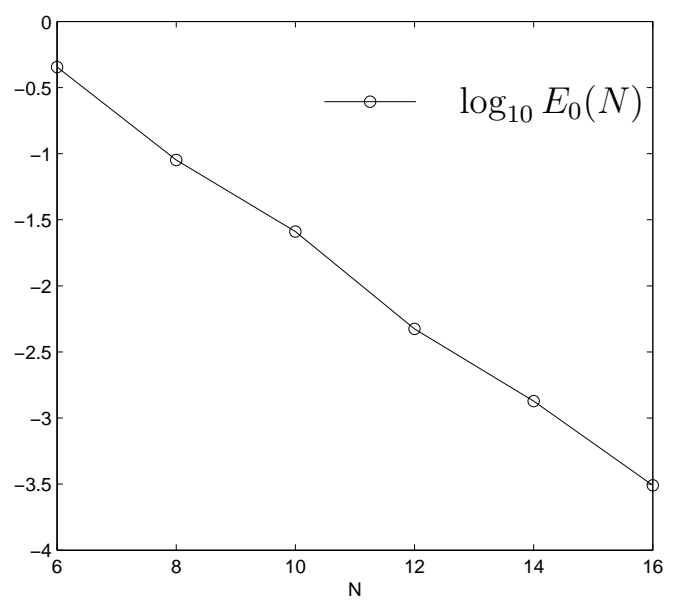

Fig. 3. Convergence test: Convergence test: $\log _{10} E_{m=0}(N)$ plotted for $N=[6, \ldots, 16], \delta^{2}=0.1$.

rotated by $45^{\circ}$ about the origin. For this large value of $\delta$, charges are located near the boundary. In each case with $\delta \ll 1$, we observed exponential convergence toward solution (32). Convergence can only be confirmed up to a limited precision since the analytic solution (32) does not correspond exactly to the problem we are solving numerically, in which sources are confined to the interior square. The best agreement can be achieved for small values of $\delta$. If the numerical solution with highest spectral resolution (here $N=64$ ) is instead taken as a reference, then the method converges to this solution spectrally up to machine precision for any value of $\delta$.

\section{Implementation}

\subsection{Summary and computation cost}

We describe the implementation of the method for our illustrative example (18) of the rectangle $[-H, H] \times[-1,1]$ with double Chebyshev discretization $(K+1) \times$ $(L+1)$.

The total preprocessing step consists of:

- Evaluation of the values and the normal derivatives of the exterior harmonic solutions on the boundary.

- Calculation of the interior harmonic solutions.

- Inversion or LU decomposition of the influence matrix,

For each particular right-hand-side $\rho$, the operations consist of: 


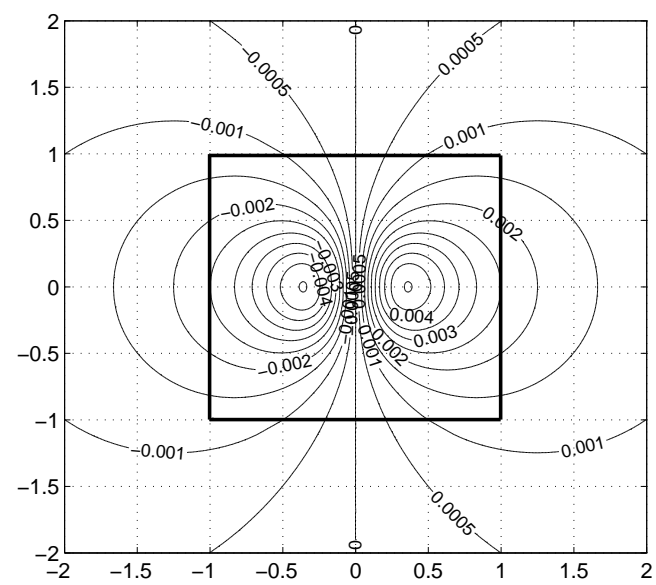

Fig. 4. Potential $\Phi_{m=1}^{N=16}$ for $\delta=0.1$.

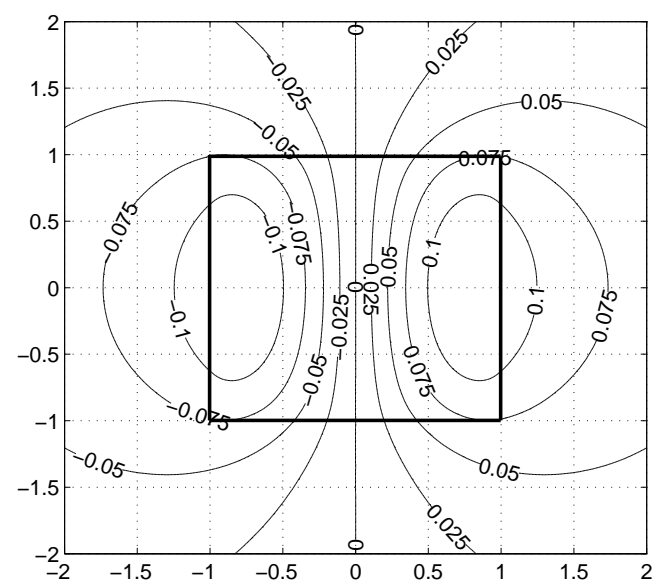

Fig. 6. Potential $\Phi_{m=1}^{N=16}$ for $\delta=2$.

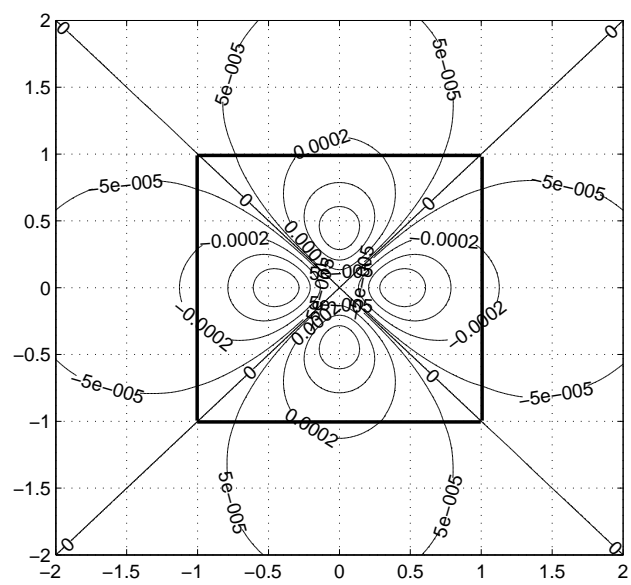

Fig. 5. Potential $\Phi_{m=2}^{N=16}$ for $\delta=0.1$.

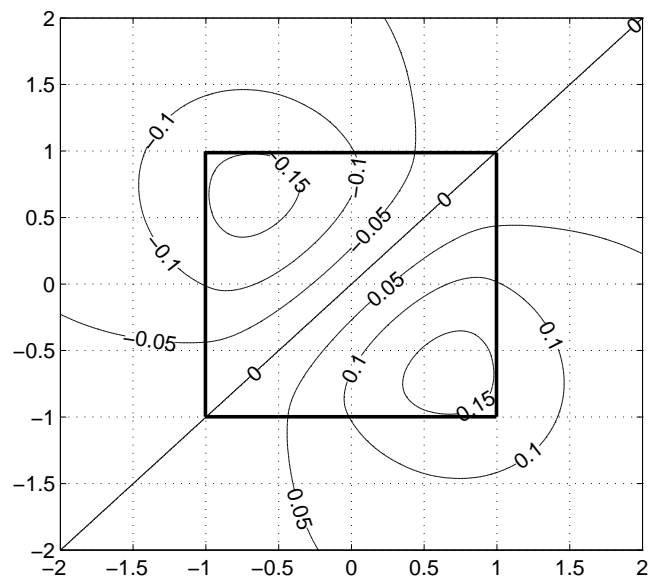

Fig. 7. $\Phi_{m=1}^{N=16}\left(r, \theta+\frac{\pi}{4}\right)$ for $\delta=2$.

- Solving for a single particular solution.

- Acting with the inverse of the influence matrix.

- Using the corrected Dirichlet boundary conditions to calculate the final solution.

The total number $J$ of boundary points is $2(K+L)$. The inversion or LU-decomposition of the influence matrix $C$ in the preprocessing stage requires a time proportional to $J^{3}$, while each solution of the linear system (15) determining the coefficients of the homogeneous solutions requires a time proportional to $J^{2}$. Each interior solution is calculated at a cost proportional to $K L^{2}$.

Symmetry can be used to reduce the cost of each step. The symmetry of the rectangle divides all the independent harmonic solutions into four mutually orthogonal 


\begin{tabular}{|l|ll|}
\hline Calculation & Result & Cost \\
\hline \multicolumn{2}{|l|}{ Preprocessing } & \multicolumn{2}{|l|}{} \\
\hline Exterior harmonic solutions & $\left.\phi_{j}\right|_{\partial \Omega},\left.\partial_{n} \phi_{j}\right|_{\partial \Omega}$ & $\begin{array}{l}K \times\left(\mathrm{Int}_{x}+\mathrm{Int}_{x}^{\prime}\right) \\
+L \times\left(\mathrm{Int}_{y}+\mathrm{Int}_{y}^{\prime}\right)\end{array}$ \\
Interior harmonic solutions & $\partial_{n} \Phi_{j}^{h}$ & $(K+L) K L^{2} / 4$ \\
Influence matrix inversion/decomposition & $C^{-1}$ & $(K+L)^{3} / 2$ \\
\hline For each right-hand-side & & \\
\hline Particular solution & $\Phi^{p}$ & $K L^{2} / 2$ \\
Action with influence matrix & $c_{j}$ & $(K+L)^{2}$ \\
Corrected solution & $\Phi$ & $K L^{2} / 2$ \\
\hline
\end{tabular}

\section{Table 1}

Operation count of each preprocessing and right-hand-side-dependent step for a rectangle discretized with $(K+1) \times(L+1)$ Chebyshev polynomials and points. Int ${ }_{x}$ and $\operatorname{Int}_{x}^{\prime}$ are the costs of performing the singular integrals over $x$ in (27) and (29), and $\operatorname{Int}_{y}$ and $\operatorname{Int}_{y}^{\prime}$ are those of the analogous integrals over $y$.

classes. Decoupling the Laplacian operator according to parity in $x$ and $y$ leads to four Poisson problems, each with resolution $K / 2 \times L / 2$, thus reducing the time by a factor of two. Decoupling by parity also reduces the influence matrix $C$ to four matrices, the dimensions of which are one fourth of that of the original matrix.

Table 5.1 gives the operation count of each step, taking into account the reductions permitted by symmetry.

\subsection{Singular integrals}

The integrations in (27)-(29) are performed numerically. Special attention must be paid in doing so since both the kernel $G\left(\mathbf{x} ; x^{\prime}\right)$ and the density $\sigma\left(x^{\prime}\right)$ have integrable singularities within the domain of integration. The singular points are $x^{\prime} \hat{\mathbf{e}}_{x}=\mathbf{x}$ and $x^{\prime}= \pm H$ for $\phi_{k}^{x}$ and $y^{\prime} \hat{\mathbf{e}}_{y}=\mathbf{x}$ and $y^{\prime}= \pm 1$ for $\phi_{l}^{y}$. Dedicated adaptive quadratures (see [34]) can be used to compute these integrals accurately.

It is also possible to evaluate the singular part of the integral analytically, reducing the numerical problem to the evaluation of integrals with non-singular integrands. The remaining integrand is piecewise $C^{\infty}$ and can be integrated with spectral precision over each of the regular subdomains. Singularity subtraction greatly decreases the variation in grid density needed to sample the integrand homogeneously, thereby significantly accelerating the numerical integrations in (27)-(29). Specifically, an adaptive method requires a smaller number of iterations, or, alternatively, a non-adaptive method requires a coarser resolution. However, the con- 


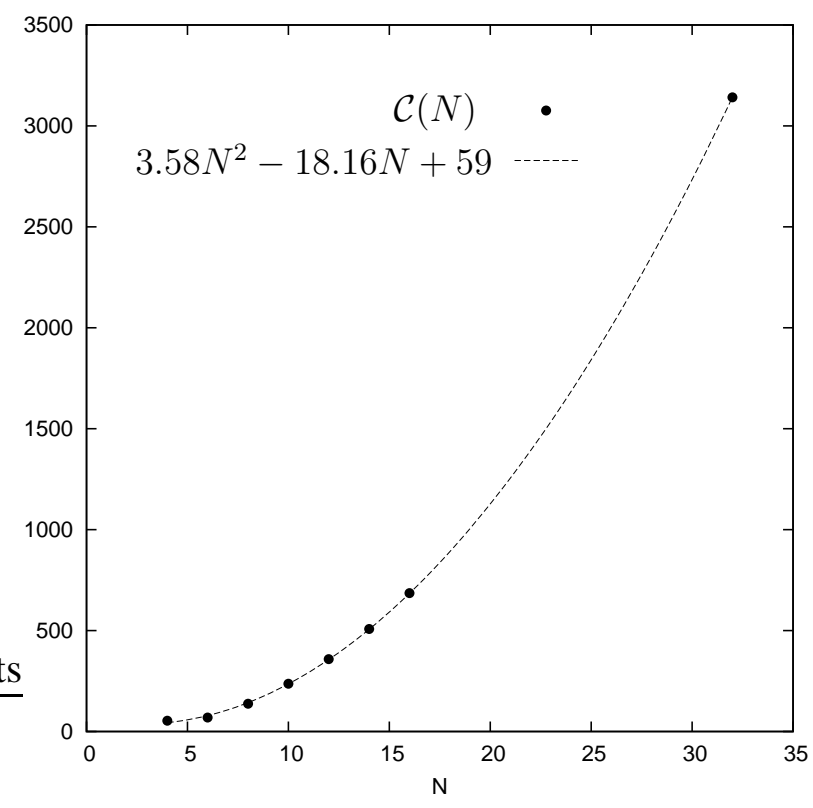

Fig. 8. Quadratic fit of the condition number $\mathcal{C}(N)$ of the influence matrix defined in (16).

vergence of our approximation with $K, L$ is exponential (spectral), regardless of whether the singular part of the integral is subtracted or included in the numerical evaluation.

\subsection{Conditioning of matrices}

The influence matrix (16) is not immediately invertible. Because of the redundancy of information at the corners, this matrix has exactly four zero singular values or eigenvalues. The corresponding linear system can be solved after arbitrarily correcting singular values or eigenvalues of the influence matrix; see [35[36] for more details.

The condition number $\mathcal{C}$ of the corrected matrix depends on the spatial resolution $N$ and the maximal order of derivatives used to express the boundary conditions. In our case of a Neumann boundary condition and a resolution $N$ in each direction, the condition number scales as $\mathcal{C}=O\left(N^{2}\right)$. Fitting the condition numbers computed for $N \in[2,32]$ with a parabola (see fig. 5.3) yields a formula for predicting the condition number for an arbitrary resolution:

$$
\mathcal{C}(N)=3.58 N^{2}-18.16 N+59
$$

It can then be deduced from (34) that a reasonably conditioned matrix with $\mathcal{C}<10^{7}$ is obtained for a spatial resolution as high as $O(1000)$. The method can therefore be applied to problems where small-scale field features require use of high spatial resolution. 


\section{Generalizations}

We now discuss the applicability of this method to other geometries, problems, and spatial discretizations. The decomposition into interior particular and homogeneous functions and exterior homogeneous functions described in section 2 is, of course, completely general and not related to any particular spatial discretization. The method described in section 3.1 for constructing the exterior harmonic functions relies on the Chebyshev-Chebyshev discretization of the rectangle which is widely used since the Chebyshev polynomials are optimal approximants of smooth functions. This property, as well as the straightfoward correspondence between interior and exterior solutions, make the Chebyshev discretization especially suitable for the construction of the exterior harmonic solutions as well. However, the method is easily generalizable to other basis functions $f_{k}(x)$ for the potential values, which can be substituted into (25) in order to calculate the corresponding charge densities $\sigma_{k}(x)$, if dictated by the geometry or numerical method used for the interior problem.

In fact, since our real interest is in generating the complete set of $\sigma_{k}(x)$ necessary to generate the complete set of $\phi_{k}(x)$, rather than in calculating the specific $\sigma_{k}(x)$ corresponding to each particular $f_{k}(x)$, the only information really required in (26) is the singularity $1 / \pi \sqrt{1-x^{2}}$. One may then allow the set of $\sigma_{k}$ 's to be the products of this singularity with the members of any appropriate basis set of analytic functions on the boundary in question.

In three dimensions, the fundamental Green's function is

$$
G\left(\mathbf{x}, \mathbf{x}^{\prime}\right)=\frac{1}{4 \pi} \frac{1}{\left|\mathbf{x}-\mathbf{x}^{\prime}\right|}
$$

In an axisymmetric geometry with a Fourier representation of the azimuthal direction, all of the problems to be solved decouple according to Fourier mode. The operation count would then scale linearly with the number of azimuthal points or Fourier modes. The elliptic problems in (6) would remain two-dimensional, and the integral equations equivalent to (24) would remain one-dimensional.

This method can also be applied to other elliptic problems or to parabolic problems. As stated in section 1, our motivation for developing this method is to apply it to the magnetohydrodynamic equations (1)-(3), in which (1) is a parabolic equation. A general parabolic problem may be written as:

$$
\partial_{t} \Phi=\Delta \Phi+\mathcal{F}(\Phi, \rho)
$$

where $\mathcal{F}$ may include nonlinear and/or time-dependent source terms. First-order implicit temporal discretization of (36) results in the inhomogeneous Helmholtz equation:

$$
(I-\delta t \Delta) \Phi(t+\delta t)=\mathcal{F}
$$


where $\mathcal{F}$ may depend on previous values of $\Phi$. This Helmholtz operator $(I-$ $\delta t \Delta)$ can replace the Laplacian in (4) and (6). It is known that replacement of the Helmholtz equation by a boundary integral equation can lead to singularities for certain values of the wavenumber (here $i / \sqrt{\delta t}$ ); a large body of work, e.g. [37/38 39:40:41] , addresses this problem. However, in the magnetohydrodynamic case of a conducting fluid surrounded by an exterior vacuum, no such difficulties would be introduced, since the exterior problem remains governed by Laplace's equation. More complicated vectorial operators may appear, as occur in the Navier-Stokes or magnetohydrodynamic equations.

\section{Comparison with other approaches}

We mention here some other techniques that have been used to solve exterior problems or to match interior and exterior domains. Spectral methods can be combined with various transformations and mappings. The inner region can be surrounded by a sphere, and the outer domain decomposed into the region inside and outside the sphere. The exterior domain can be mapped into an interior domain via a $1 / r$ mapping [6 42 43]; spectral methods can then be used to treat either or both domains. The region exterior to one or more spheres has been mapped to the interior of a rectangle [44] or a pentangular [45] region rotated about an axis, and ChebyshevFourier expansions used to solve elliptic equations arising in the study of black holes in general relativity. A smooth boundary can be parameterized by angle, and the boundary values represented as a series of trigonometric functions or spherical harmonics [22 39404142 43:46/47/48]. Our method differs from these in that a Chebyshev approximation is used to represent the boundary values on each segment of a non-smooth boundary, and an analytic formula is used to calculate the surface density which exactly yields this Chebyshev approximation.

Conformal mapping is another technique which can be used to calculate interior or exterior harmonic functions. The Riemann mapping theorem guarantees the existence of a conformal transformation from the interior or exterior of a simply connected domain into the interior or exterior of a unit disk; its proof is, however, non-constructive, and does not explicitly derive the transformation. For some geometries, including the exterior of a rectangle, an analytical formula can be derived [49]. For polygon-bounded regions with piecewise-constant boundary conditions, the Schwarz-Christoffel [50] mapping has proved to be a very robust tool, applied to problems arising in magneto- and electro- statics, potential flows, inverse problems and many other fields.

Our influence matrix approach relies on calculating harmonic functions with arbitrary boundary data, for which conformal mapping is much more problematic. More general conformal mappings are often computed by solving Symm's or Carleman's equation (24) numerically on the domain boundary, making this approach 
similar in terms of numerical cost and precision to boundary integral equation methods. It is interesting to note that, for domains including corners, the Chebyshev approximation is especially well suited, guaranteeing superconvergence of the mapping function [33].

\section{Conclusion}

As a test case for the magnetohydrodynamic equations, we have developed a method for solving the two-dimensional Poisson equation in a bounded domain, where the solution satisfies matching conditions with a harmonic potential outside the domain. The method solves only the interior problem and determines the boundary conditions ensuring smooth matching with the exterior solution. The essential element of this approach is construction of a basis of harmonic functions which represent the near-boundary exterior solutions uniformly. This basis is used to construct the influence matrix which serves to impose the coupled boundary conditions between the interior and exterior solutions. The method is numerically reasonably well conditioned and can be used for quite high spatial resolutions. For a spectral solver, this method guarantees exponential convergence.

Instead of corresponding to point sources on the boundary, each exterior harmonic solution corresponds to a spectral basis function. The most costly process - the construction of a basis of exterior harmonic functions - depends only on geometry and spatial resolution. Once the basis is computed it can be stored and used for any computation using the same resolution and domain shape. When used as a preprocessing step for time-dependent simulations, the cost of constructing the exterior harmonic basis is negligible compared to that of thousands of time steps.

Since cylindrical coordinates have one periodic direction, it should be possible to apply this method separately to each of the Fourier modes, treated individually as two-dimensional problems. The extension of this method to the MHD equations in a finite cylindrical geometry is currently under investigation.

\section{References}

[1] R. Steglitz \& U. MÜLleR, Experimental demonstration of a homogenous twoscale dynamo, Magnetohydrodynamics 38, 27 (2002).

[2] A. Gailitis, O. Lielaisis, E. Platacis, S. Dementev, A. Cifersons, G. Gerbeth, T. Gundrum, F. Stefani, M. Christen \& G. Will, Magnetic field saturation in the Riga dynamo experiment, Phys. Rev. Lett. 86, 3024 (2001).

[3] M. Bourgoin, L. Marié, F. Petrelis, C. Gasquet, A. Guigon, J.B. Luciani, M. Mulin, F. Namer, J. Burgete, A. Chiffaudel, F. Daviaud, S. Fauve, 
P. ODIER \& J.F. PINTON Magnetohydrodynamics measurements in the von Kármán sodium experiment, Phys. Fluids 14, 3046 (2002).

[4] C.B. Forest, R.A. BAyliss, R.D. Kendrick, M.D. Nornberg, R. O'CONNELl \& E.J. SPENCE, Hydrodynamic and numerical modeling of a spherical homogeneous dynamo experiment, Magnetohydrodynamics 38, 107 (2002).

[5] W.L. Shew, D.R. Sisan, \& D.P. LAThrop, Mechanically forced and thermally driven flows in liquid sodium, Magnetohydrodynamics 38, 121 (2002).

[6] S.A. JEPPS, Numerical models of hydromagnetic dynamos, J. Fluid Mech. 67, 625 (1967).

[7] M. Dudley \& R. James, Time-dependent kinematic dynamos with stationary flows, Proc. Roy. Soc. London A 425, 407-429 (1989).

[8] G. A. Glatzmaier and P. H. Roberts A three-dimensional self-consistent computer simulation of a geomagnetic field reversal, Nature 337, 203 (1995).

[9] A. Tilgner, A kinematic dynamo with a small scale velocity field, Phys. Rev. A 226, 75-79 (1997).

[10] R. Hollerbach, A spectral solution of the magneto-convection equations in spherical geometry, Int. J. for Num. Meth. in Fluids 32, 773-797 (2000).

[11] A. P. Willis \& C.F. Barenghi, A Taylor-Couette Dynamo, Astronomy \& Astrophysics 393, 339-343 (2002).

[12] H. Matsui \& H. OKuda, Development of a simulation code for MHD dynamo processes using the GeoFEM platform, Intl. J. of Comp. Fluid Dyn. 18, 323-332 (2004).

[13] A. B. Iskakov, S. Descombes \& E. Dormy, An integro-differential formulation for magnetic induction in bounded domains: boundary element-finite volume method, J. Comput. Phys. 7, 540-554 (2004).

[14] A. B. Iskakov \& E. DORMY, On magnetic boundary conditions for non-spectral dynamo simulations, Geophys. Astrophys. Fluid Dyn. 99, 481-492 (2005).

[15] M. Xu, F. Stefani \& G. Gerbeth, The integral equation method for a steady kinematic dynamo problem, J. Comput. Phys. 196, 102-125 (2004).

[16] J.L. Guermond, R. Laguerre, J. Léorat \& C. Nore, An interior penalty Galerkin method for the MHD equations in heterogeneous domains, J. Comput. Phys. 221, 349-369 (2007).

[17] P. Roberts, An Introduction to Magnetohydrodynamics, Longmans Green, London, 1967.

[18] G. Hsiao \& R.C. MACCAmy Solution of Boundary Value Problems by Integral Equations of the First Kind SIAM Review 15, 687-705 (1973).

[19] M.A. JASWON \& G.T. SYMM, Integral equation methods in potential theory and elastostatics, Academic Press, 1977. 
[20] C. Brebbia \& S. WalkeR, The boundary element method for engineers, Pentech Press, 1978.

[21] P.K. BANerJeE \& R. Butterfield, Boundary element methods in engineering science, McGraw-Hill, 1981.

[22] K. E. AtKinson, The numerical solution of Laplace's equation in three dimensions, SIAM J. Num. Anal. 19, 263-274 (82).

[23] V. Rokhlin, Rapid solution of integral equations of classical potential theory, J. Comput. Phys. 60, 187-207 (1983).

[24] C.A. Brebbia, T. Futagami \& M. Tanaka, EdS., Boundary Elements: Proceedings of the 5th International Conference, Springer, 1983.

[25] C. PozRIKIDIS, Boundary integral and singularity methods for linearized viscous flow, Cambridge Univ. Press, 1992.

[26] S. KIRKUP, http://www.boundary-element-method.com

[27] S.A. ORSZAG, Numerical simulation of incompressible flows within simple boundaries: I. Galerkin (spectral) representations, Stud. Appl. Math. 50, 293-327 (1971).

[28] C. Canuto, M.Y. Hussaini, A. Quateroni \& T.A. Zang, Spectral Methods in Fluid Dynamics, Springer, 1988.

[29] K. JÖRGENS, Integral operators, Pitman, Boston, 1982. Translation from German of Lineare Integraloperatoren, Teubner, Stuttgart, 1970.

[30] A. D. Polyanin \& A. V. MAnZhirov, Handbook of Integral Equations, CRC Press, Boca Raton, 1998.

[31] J.C. MASOn \& D.C. HAndscomb, Chebyshev Polynomials, Chapman \& Hall/CRC Press, 2000.

[32] L. N. Trefethen, Spectral Methods in Matlab (SIAM, Philadelphia, 2000).

[33] J. Levesley, D. M. Hough \& S.N. ChandleR-Wilde, A Chebyshev collocation method for solving Symm's integral equation for conformal mapping: a partial error analysis, IMA J. Num. Anal. 14, 57-79 (1993).

[34] W.H. Press, B.P. Flannery, S.A. Teukolsky \& W.T. Vetterling, Numerical Recipes: The Art of Scientific Computing, Cambridge Univ. Press, 1986.

[35] L.S. Tuckerman, Divergence-free velocity fields in nonperiodic geometries, J. Comput. Phys. 80, 403-441 (1989).

[36] P. Boronski, A Method Based on Poloidal-Toroidal Potentials Applied to the von Kármán Flow in a Finite Cylinder Geometry, Ph.D. Thesis, Ecole Polytechnique, 2005.

[37] S. AMINi \& S.M. KIRKUP, Solution of Helmholtz equation in the exterior domain by elementary boundary integral methods, J. Comput. Phys. 118, 208-221 (1995). 
[38] D. Givoli \& I. Harari, eds., Special Issue on Exterior Problems of Wave Propagation, Comput. Methods Appl. Mech. Engrg. 164, 1-266 (1998).

[39] K. Gerdes \& L. Demkowicz, Solution of 3D-Laplace and Helmholtz equations in exterior domains using hp-infinite elements, Comput. Methods Appl. Mech. Engrg. 137, 239-272 (1996).

[40] W.S. HWANG, A boundary spectral method for solving exterior acoustical problems with hypersingular integrals, Int. J. Numer. Meth. Engng. 44, 1775-1783 (1999).

[41] T.-C. Lin \& Y. WARnAPAla-YehiYA, The numerical solution of exterior Neumann problem for Helmholtz's equation via modified Green's functions approach, Computers and Mathematics with Applications 47, 593-609 (2004).

[42] P. Grandclement, S. Bonazzola, E. Gourgoulhon \& J.-A. Marck, A multidomain spectral method for scalar and vectorial Poisson equations with noncompact sources, J. Comput. Phys. 170, 231-260 (2001).

[43] M.-C. LAI, Z. LI \& X. LIN, Fast solvers for 3D Poisson equations involving interfaces in a finite or the infinite domain, J. Comput. Appl. Math. 181, 106-125 (2006).

[44] M. Ansorg, B. Brügmann \& W. TIChy, A single-domain spectral method for black hole puncture data, Phys. Rev. D 70, 064011 (2004).

[45] M. Ansorg, Double-domain spectral method for black hole excision data, Phys. Rev. D 72, 024018 (2005).

[46] S. Meddahi \& A. Márquez, A combination of spectral and finite elements for an exterior problem on the plane, Appl. Numer. Math. 43, 275-295 (2002).

[47] Y. CHEN, Galerkin methods for solving single layer integral equations in three dimensions, Ph.D. Thesis, Univ. of Iowa, 1994.

[48] M. Ganesh, I.G. Graham \& J. Sivaloganathan, A new spectral boundary integral collocation method for three-dimensional potential problems, SIAM J. Num. Anal. 35, 778-805 (1998).

[49] V.I. Ivanov \& M.K. Trubetskov, Handbook of Conformal Mapping with Computer-Aided Visualization, CRC Press, Boca Raton, 1995.

[50] T.A. Driscoll \& L.N. Trefethen, Schwarz-Christoffel Mapping, Cambridge Univ. Press, 2002. 